\title{
Homoclinic solutions for second order Hamiltonian systems near the origin
}

\section{Li-Li Wan*}

${ }^{*}$ Correspondence:

15882872311@163.com

School of Science, Southwest

University of Science and

Technology, Mianyang, Sichuan

621010, China

\begin{abstract}
Under some local superquadratic conditions on $W(t, u)$ with respect to $u$, the existence of infinitely many homoclinic solutions is obtained for the nonperiodic second order Hamiltonian systems $\ddot{u}(t)-L(t) u(t)+\nabla W(t, u(t))=0, \forall t \in \mathbb{R}$, where $L(t)$ is unnecessarily coercive.
\end{abstract}

Keywords: homoclinic solutions; second order Hamiltonian systems; local conditions

\section{Introduction and main results}

Let us consider the second order Hamiltonian systems

$$
\ddot{u}(t)-L(t) u+\nabla W(t, u(t))=0, \quad \forall t \in \mathbb{R},
$$

where $L \in C\left(\mathbb{R}, \mathbb{R}^{N^{2}}\right)$ is a symmetric matrix-valued function and $\nabla W(t, x)=\frac{\partial}{\partial x} W(t, x)$. As usual, we say that $u$ is a nontrivial homoclinic solution (to 0 ) if $u \in C^{2}\left(\mathbb{R}, \mathbb{R}^{N}\right), u \neq 0$, $u(t) \rightarrow 0$ as $|t| \rightarrow \infty$. In the following, (., $\cdot): \mathbb{R}^{N} \times \mathbb{R}^{N} \mapsto \mathbb{R}$ denotes the standard inner product in $\mathbb{R}^{N}$ and $|\cdot|$ is the induced norm, and let $I_{N}$ be the identity matrix of order $N$.

With the variational methods, the existence and multiplicity of homoclinic orbits of problem (1) have been obtained in many papers (see [1-16]), mainly in the case that $W$ satisfies some global assumptions for all $t$ and $u$. Among other results, under some local conditions on $W, \mathrm{Lv}$ and Jiang [6] investigated the existence of one nontrivial homoclinic solution for the second order Hamiltonian systems

$$
\ddot{u}-L(t) u(t)+\nabla W(t, u(t))=f(t), \quad \forall t \in \mathbb{R}
$$

as a limit of periodic solutions of a certain sequence of boundary-value problems. Later, it was proved in [16] that if $L(t)$ is coercive and $W(t, u)$ is subquadratic near the origin with respect to $u$, then problem (1) has a sequence of homoclinic solutions converging to zero in $L^{\infty}$ norm. There were no conditions assumed on $W$ for $u$ large. More precisely, one presented the following assumptions:

$\left(\mathrm{A}_{1}\right)$ There exists a constant $\alpha<2$ such that $l(t)|t|^{\alpha-2} \rightarrow \infty$ as $|t| \rightarrow \infty$, where

$$
l(t)=\inf _{x \in \mathbb{R}^{N},|x|=1}(L(t) x, x) .
$$

(c) 2016 Wan. This article is distributed under the terms of the Creative Commons Attribution 4.0 International License (http://creativecommons.org/licenses/by/4.0/), which permits unrestricted use, distribution, and reproduction in any medium, provided you give appropriate credit to the original author(s) and the source, provide a link to the Creative Commons license, and indicate if changes were made. 
$\left(\mathrm{A}_{2}\right)$ There are constants $c_{0}>0$ and $\frac{1}{2} \leq v \in\left(\frac{1}{3-\alpha}, 1\right)$ such that

$$
|\nabla W(t, x)| \leq c_{0}|x|^{\nu}, \quad \forall(t, x) \in \mathbb{R} \times B_{\delta}(0)
$$

where $B_{\delta}(0)$ denotes the ball in $\mathbb{R}^{N}$ centered at 0 with radius $\delta>0$.

$\left(\mathrm{A}_{3}\right)$

$$
\lim _{|x| \rightarrow 0} \frac{W(t, x)}{|x|^{2}}=\infty \quad \text { uniformly for } t \in \mathbb{R}
$$

$\left(\mathrm{A}_{4}\right) 2 W(t, x)-(\nabla W(t, x), x)>0$ for all $t \in \mathbb{R}$ and $x \in \mathbb{R}^{N} \backslash\{0\}$.

In this note, we will consider problem (1) where $L(t)$ is unnecessarily coercive, and $W(t, u)$ is superquadratic near the origin. The exact assumptions on $L$ and $W$ are as follows.

Theorem 1 Assume the following conditions hold:

$\left(\mathrm{L}_{1}\right) \quad$ There exists $l_{0} \geq 0$ such that

$$
l(t):=\inf _{x \in \mathbb{R}^{N},|x|=1}(L(t) x, x) \geq-l_{0}, \quad \forall t \in \mathbb{R} .
$$

$\left(\mathrm{L}_{2}\right) \quad$ There exists a constant $\xi>1$ such that

$$
\operatorname{meas}\left(\left.t \in \mathbb{R}|| t\right|^{-\xi} L(t) \ngtr M_{0} I_{N}\right)<+\infty, \quad \forall M_{0}>0 .
$$

$\left(\mathrm{W}_{1}\right) \quad W \in C^{1}\left(\mathbb{R} \times B_{\delta}(0), \mathbb{R}\right)$ is even in $u$ and $W(t, 0)=0$, where $B_{\delta}(0)$ denotes the ball in $\mathbb{R}^{N}$ centered at 0 with radius $\delta>0$.

$\left(\mathrm{W}_{2}\right)$ There are constants $c_{1}>0$ and $0<\theta<1$ such that

$$
|\nabla W(t, x)| \leq c_{1}|x|^{\theta}, \quad \forall(t, x) \in \mathbb{R} \times B_{\delta}(0) .
$$

$\left(\mathrm{W}_{3}\right)$ There exists a constant $p>2$ such that

$$
\lim _{|x| \rightarrow 0} \frac{W(t, x)}{|x|^{p}}=0 \quad \text { uniformly for } t \in \mathbb{R} .
$$

$\left(W_{4}\right) 2 W(t, x)-(\nabla W(t, x), x)<0$ for all $t \in \mathbb{R}$ and $x \in \mathbb{R}^{N} \backslash\{0\}$.

$\left(\mathrm{W}_{5}\right)$ There exists a constant $\mu>2$ such that

$$
\lim _{|x| \rightarrow 0} \frac{W(t, x)}{|x|^{\mu}}=\infty \quad \text { uniformly for } t \in \mathbb{R}
$$

Then problem (1) has a sequence of homoclinic solutions $\left\{u_{k}\right\}$ such that $\max _{t \in \mathbb{R}}\left|u_{k}(t)\right| \rightarrow 0$ as $k \rightarrow \infty$.

Remark 1 There exist $L$ and $W$ that satisfy all assumptions in Theorem 1 . For example, let $L(t)=\left(t^{4} \sin ^{2} t+1\right) I_{N}$ and $W(t, x)=|x|^{4}$ for $|x|<1$ with $\theta=\frac{1}{2}, p=3, \mu=5$. Note that since $L$ does not satisfy the coercive condition $\left(\mathrm{A}_{1}\right)$ and $W$ is superquadratic near the origin, 
Theorem 1 is different from Theorem 1.1 in [16]. As far as the authors know, there is little research concerning the multiplicity of homoclinic solutions for problem (1) simultaneously under local conditions and noncoercive conditions, so our result is different from the previous results in the literature.

The proof is motivated by the argument in [16]. We will modify and extend $W$ to an appropriate $\widetilde{W}$ and show for the associated modified functional $I$ the existence of a sequence of homoclinic solutions converging to zero in $L^{\infty}$ norm, and therefore we obtain infinitely many homoclinic solutions for the original problem.

\section{Proof of theorems}

First of all, we introduce the Sobolev space that we study. Let $A$ be the self-adjoint extension of the operator $-\left(\frac{d^{2}}{d t^{2}}\right)+L(t)$ with the domain $\mathcal{D}(A) \subset L^{2} \equiv L^{2}\left(\mathbb{R}, \mathbb{R}^{N}\right)$. Denote by $\{E(\lambda) \mid-\infty<\lambda<\infty\}$ and $|A|$ the spectral resolution and the absolute value of $A$, respectively. Define $U=I-E(0)-E(-0)$. $U$ commutes with $A,|A|$ and $|A|^{1 / 2}$, and $A=U|A|$ is the polar decomposition of $A$ (see [17]). Let $E=\mathcal{D}\left(|A|^{1 / 2}\right)$, the domain of $|A|^{1 / 2}$. Define on $E$ the inner product and the corresponding norm:

$$
\begin{aligned}
& (u, v)_{0}=\left(|A|^{1 / 2} u,|A|^{1 / 2} v\right)_{2}+(u, v)_{2}, \\
& \|u\|_{0}=(u, u)_{0}^{1 / 2}
\end{aligned}
$$

where $u, v \in E$ and $(\cdot, \cdot)_{2}$ denotes the inner product of $L^{2}$. Then $E$ is a Hilbert space, and it is easy to verify that $E$ is continuously embedded in $H^{1}\left(\mathbb{R}, \mathbb{R}^{N}\right)$.

Lemma 1 (see [10]) Suppose that $L(t)$ satisfies $\left(\mathrm{L}_{1}\right)$ and $\left(\mathrm{L}_{2}\right)$. Then $E$ is compactly embedded in $L^{q}\left(\mathbb{R}, \mathbb{R}^{N}\right)$ for $q \in[1, \infty]$.

Remark 2 By Lemma 1 it is easy to prove that the spectrum $\sigma(A)$ consists of eigenvalues numbered by $\lambda_{1} \leq \lambda_{2} \leq \cdots \rightarrow+\infty$, and a corresponding system of eigenfunctions, $\left(e_{j}\right)\left(A e_{j}=\lambda_{j} e_{j}\right)$, forms an orthogonal basis in $L^{2}$. Let $j^{-}=\#\left\{j \mid \lambda_{j}<0\right\}, j^{0}=\#\left\{j \mid \lambda_{j}=0\right\}$ and $\bar{j}=j^{-}+j^{0}$, where $\# \mathcal{A}$ denotes the number of elements of the set $\mathcal{A}$. Set $E^{-}=\operatorname{span}\left\{e_{1}, \ldots, e_{j^{-}}\right\}$, $E^{0}=\operatorname{span}\left\{e_{j^{-}+1}, \ldots, e_{j}\right\}=\operatorname{ker} A$, and $E^{+}=\overline{\operatorname{span}\left\{e_{j_{+1}}, \ldots\right\}}$. Then one has $E=E^{-} \oplus E^{0} \oplus E^{+}$. We introduce on $E$ the following inner product and the corresponding norm:

$$
\begin{aligned}
& (u, v)=\left(|A|^{\frac{1}{2}} u,|A|^{\frac{1}{2}} v\right)_{2}+\left(u^{0}, v^{0}\right)_{2}, \\
& \|u\|^{2}=(u, u)=\left\||A|^{\frac{1}{2}} u\right\|_{2}^{2}+\left\|u^{0}\right\|_{2}^{2},
\end{aligned}
$$

where $u=u^{-}+u^{0}+u^{+}$and $v=v^{-}+v^{0}+v^{+} \in E=E^{-} \oplus E^{0} \oplus E^{+}$. Obviously the norms $\|\cdot\|$ and $\|\cdot\|_{0}$ are equivalent and so the norm $\|\cdot\|$ on $E$ will always be used. By Lemma 1 we see that there exists a constant $\gamma_{q}>0$ such that

$$
\|u\|_{q} \leq \gamma_{q}\|u\|, \quad \forall u \in E, \forall q \in[1, \infty]
$$

Lemma 2 Assume that $\left(\mathrm{W}_{1}\right)-\left(\mathrm{W}_{4}\right)$ are satisfied. There is $0<r<\frac{\delta}{2}$ and $\widetilde{W} \in C^{1}\left(\mathbb{R}, \mathbb{R}^{N}\right)$ such that 
(i)

$$
|\nabla \widetilde{W}(t, x)| \leq c_{2}\left(|x|^{\theta}+|x|^{p-1}\right), \quad \forall(t, x) \in \mathbb{R} \times \mathbb{R}^{N},
$$

where $c_{2}$ is a constant;

(ii)

$$
\widehat{W}(t, x):=2 \widetilde{W}(t, x)-(\nabla \widetilde{W}(t, x), x) \leq 0, \quad \forall(t, x) \in \mathbb{R} \times \mathbb{R}^{N}
$$

and

$$
\widehat{W}(t, x)=0 \quad \text { iff } \quad|x|=0 .
$$

Proof By the mean value theorem, $\left(\mathrm{W}_{1}\right)$ and $\left(\mathrm{W}_{2}\right)$ imply that

$$
|W(t, x)| \leq c_{1}|x|^{\theta+1}, \quad \forall(t, x) \in \mathbb{R} \times B_{\delta}(0) .
$$

Next we modify $W(t, x)$ for $x$ outside a neighborhood of the origin 0 . Choose

$$
0<\beta<\frac{1}{4 \gamma_{p}^{p}}
$$

where $\gamma_{p}$ is the constant given in (2). By $\left(\mathrm{W}_{3}\right)$, there is a constant $r \in\left(0, \frac{\delta}{2}\right)$ such that

$$
W(t, x) \leq \beta|x|^{p}, \quad \forall t \in \mathbb{R} \text { and }|x| \leq 2 r .
$$

Define a cut-off function $\rho \in C^{1}(\mathbb{R}, \mathbb{R})$ satisfying

$$
\rho(t)= \begin{cases}1, & 0 \leq t \leq r \\ 0, & t \geq 2 r\end{cases}
$$

and $-\frac{2}{r} \leq \rho^{\prime}(t)<0$ for $r<t<2 r$. Using $\rho$, we define

$$
\widetilde{W}(t, x):=\rho(|x|) W(t, x)+(1-\rho(|x|)) W_{\infty}(x), \quad \forall(t, x) \in \mathbb{R} \times \mathbb{R}^{N},
$$

where $W_{\infty}(x)=\beta|x|^{p}$. Then by direct computation we get

$$
\begin{aligned}
\nabla \widetilde{W}(t, x) & =\rho(|x|) \nabla W(t, x)+\rho^{\prime}(|x|) W(t, x)+(1-\rho(|x|)) W_{\infty}^{\prime}(x)-\rho^{\prime}(|x|) W_{\infty}(x), \\
\widehat{W}(t, x)= & \rho(|x|)(2 W(t, x)-(\nabla W(t, x), x))+(2-p)(1-\rho(|x|)) W_{\infty}(x) \\
& -\rho^{\prime}(|x|)\left(W(t, x)-W_{\infty}(x)\right)|x|
\end{aligned}
$$

for $(t, x) \in \mathbb{R} \times \mathbb{R}^{N}$. It follows from $\left(\mathrm{W}_{1}\right)$ and $\left(\mathrm{W}_{2}\right)$ that

$$
\nabla \widetilde{W}(t, 0)=\widehat{W}(t, 0)=0, \quad \forall t \in \mathbb{R} .
$$

Then by (6), (9), $\left(\mathrm{W}_{2}\right)$, and the choice of the cut-off function $\rho$, we have

$$
|\nabla \widetilde{W}(t, x)| \leq \beta p|x|^{p-1}, \quad \forall t \in \mathbb{R},|x| \geq 2 r
$$


and

$$
\begin{aligned}
|\nabla \widetilde{W}(t, x)| & \leq|\nabla W(t, x)|+\frac{2}{r}|W(t, x)|+W_{\infty}^{\prime}(x)+\frac{2}{r} W_{\infty}(x) \\
& \leq c_{1}|x|^{\theta}+4 c_{1}|x|^{\theta}+\beta p|x|^{p-1}+4 \beta|x|^{p-1} \\
& =5 c_{1}|x|^{\theta}+(4+p) \beta|x|^{p-1}, \quad \forall t \in \mathbb{R},|x|<2 r .
\end{aligned}
$$

Therefore, (3) is satisfied if $c_{2}=\max \left\{5 c_{1},(4+p) \beta\right\}$.

Finally, we prove (4) and (5). On one hand, using (11) we know that $\widehat{W}(t, x)=0$ whenever $x=0$. On the other hand, assume that $r<|x|<2 r$. By (10), $\left(\mathrm{W}_{4}\right)$, (7), and the choice of the cut-off function $\rho$, we obtain

$$
\begin{aligned}
& \rho(|x|)(2 W(t, x)-(\nabla W(t, x), x))<0 \\
& (2-p)(1-\rho(|x|)) W_{\infty}(x) \leq 0
\end{aligned}
$$

and

$$
-\rho^{\prime}(|x|)\left(W(t, x)-W_{\infty}(x)\right)|x| \leq 0 .
$$

The above estimates imply that $\widehat{W}(t, x)<0$ if $r<|x|<2 r$. Besides, when $|x| \geq 2 r$, by (10) we have

$$
\widehat{W}(t, x)=(2-p) W_{\infty}(x)<0 .
$$

When $0<|x| \leq r$, by $\left(\mathrm{W}_{4}\right)$ we get

$$
\widehat{W}(t, x)=2 W(t, x)-(\nabla W(t, x), x)<0
$$

Thus (4) and (5) are verified. The proof is completed.

We now consider the modified problem

$$
\ddot{u}(t)-L(t) u+\nabla \widetilde{W}(t, u(t))=0, \quad \forall t \in \mathbb{R},
$$

whose solutions correspond to critical points of the functional

$$
\begin{aligned}
I(u) & =\frac{1}{2} \int_{\mathbb{R}}\left(|\dot{u}|^{2}+(L(t) u, u)\right) d t-\int_{\mathbb{R}} \widetilde{W}(t, u) d t \\
& =\frac{1}{2}\left\|u^{+}\right\|^{2}-\frac{1}{2}\left\|u^{-}\right\|^{2}-\int_{\mathbb{R}} \widetilde{W}(t, u) d t
\end{aligned}
$$

for all $u=u^{-}+u^{0}+u^{+} \in E=E^{-}+E^{0}+E^{+}$. By (6) and (8) we have

$$
|\widetilde{W}(t, u)| \leq c_{1}|u|^{\theta+1}+\beta|u|^{p}, \quad \forall(t, u) \in \mathbb{R} \times \mathbb{R}^{N}
$$

Thus, $I$ is well defined. 
Rewrite $I$ as follows:

$$
I=I_{1}-I_{2}
$$

where

$$
I_{1}=\frac{1}{2} \int_{\mathbb{R}}\left(|\dot{u}|^{2}+(L(t) u, u)\right) d t \quad \text { and } \quad I_{2}=\int_{\mathbb{R}} \widetilde{W}(t, u) d t .
$$

In the following, $c$ will be used to denote various positive constants where the exact values are different.

Lemma 3 Let $\left(\mathrm{L}_{1}\right),\left(\mathrm{L}_{2}\right),\left(\mathrm{W}_{1}\right)$ and $\left(\mathrm{W}_{2}\right)$ be satisfied. Then $I_{2} \in C^{1}(E, \mathbb{R})$ and $I_{2}^{\prime}: E \rightarrow E^{*}$ is compact, and hence $I \in C^{1}(E, \mathbb{R})$. Moreover,

$$
\begin{aligned}
\left\langle I_{2}^{\prime}(u), v\right\rangle & =\int_{\mathbb{R}}(\nabla \widetilde{W}(t, u), v) d t \\
\left\langle I^{\prime}(u), v\right\rangle & =\left(u^{+}, v^{+}\right)-\left(u^{-}, v^{-}\right)-\int_{\mathbb{R}}(\nabla \widetilde{W}(t, u), v) d t
\end{aligned}
$$

for $u, v \in E=E^{-} \oplus E^{0} \oplus E^{+}$, and nontrivial critical points of $I$ on $E$ belong to $C^{2}\left(\mathbb{R}, \mathbb{R}^{N}\right)$ and are homoclinic solutions of problem (12).

Proof By (3), for any $\eta \in[0,1], u, h \in \mathbb{R}^{N}$ we have

$$
|(\nabla \widetilde{W}(t, u+\eta h), h)| \leq c\left(|u|^{\theta}|h|+|h|^{\theta+1}+|u|^{p-1}|h|+|h|^{p}\right),
$$

where $c$ is independent of $\eta$. Hence, for any $u, h \in E$, by the mean value theorem and Lebesgue's dominated convergence theorem, we get

$$
\begin{aligned}
\lim _{s \rightarrow 0} \frac{I_{2}(u+s h)-I_{2}(h)}{s} & =\lim _{s \rightarrow 0} \int_{\mathbb{R}}(\nabla \widetilde{W}(t, u+\tau(t) s h), h) d t \\
& =\int_{\mathbb{R}}(\nabla \widetilde{W}(t, u), h) d t \\
& :=W_{0}(u, h),
\end{aligned}
$$

where $\tau(t) \in[0,1]$ depends on $u, h, s$. Moreover, it follows from (2) and (3) that

$$
\begin{aligned}
\left|W_{0}(u, h)\right| & \leq \int_{\mathbb{R}}|(\nabla \widetilde{W}(t, u), h)| d t \\
& \leq c\left(\|u\|_{\theta+1}^{\theta}\|h\|_{\theta+1}+\|u\|_{p}^{p-1}\|h\|_{p}\right) \\
& \leq c\left(\|u\|^{\theta}+\|u\|^{p-1}\right)\|h\| .
\end{aligned}
$$

Therefore, $W_{0}(u, \cdot)$ is linear and bounded in $h$, and $d I_{2}(u)=W_{0}(u, \cdot) \in E^{*}$ is the Gateaux derivative of $I_{2}$ at $u$.

Next we prove that $d I_{2}(u)$ is weakly continuous. Set $B u:=\nabla \widetilde{W}(t, u)$. There exist $B_{1}, B_{2}$ such that $B=B_{1}+B_{2}$, where $B_{1}$ is bounded and continuous from $L^{\theta+1}(\mathbb{R})$ to $L^{\frac{\theta+1}{\theta}}(\mathbb{R})$ and 
$B_{2}$ is bounded and continuous from $L^{p}(\mathbb{R})$ to $L^{\frac{p}{p-1}}(\mathbb{R})$. For any $v, h \in E$,

$$
\begin{aligned}
\left|\left\langle d I_{2}(u)-d I_{2}(v), h\right)\right| & =\left|\int_{\mathbb{R}}(B u-B v, h) d t\right| \\
& =\left|\int_{\mathbb{R}}\left(B_{1} u+B_{2} u-B_{1} v-B_{2} v, h\right) d t\right| \\
& \leq \int_{\mathbb{R}}\left|B_{1} u-B_{1} v\right||h| d t+\int_{\mathbb{R}}\left|B_{2} u-B_{2} v \| h\right| d t \\
& \leq c\left\|B_{1} u-B_{1} v\right\|_{\frac{\theta+1}{\theta}}\|h\|+c\left\|B_{2} u-B_{2} v\right\|_{\frac{p}{p-1}}\|h\|,
\end{aligned}
$$

which implies that

$$
\left\|d I_{2}(u)-d I_{2}(v)\right\|_{E^{*}} \leq c\left\|B_{1} u-B_{2} v\right\|_{\frac{\theta+1}{\theta}}+c\left\|B_{2} u-B_{2} v\right\|_{\frac{p}{p-1}} .
$$

Now suppose $u_{n} \rightarrow u$ in $E$, then by Lemma $1, u_{n} \rightarrow u$ in $L^{(\theta+1)}(\mathbb{R})$ and $L^{p}(\mathbb{R})$. Combining the above arguments, we see that $d I_{2}(u)$ is weakly continuous. Therefore, $I_{2}(u) \in C^{1}(E, \mathbb{R})$ and $I_{2}^{\prime}: E \rightarrow E^{*}$ is compact.

Finally, we show that nontrivial critical points of $I$ on $E$ are homoclinic solutions of problem (12). Let $u \in E$ be a nontrivial critical point of $I$. A standard argument shows that $u \in C^{2}\left(\mathbb{R}, \mathbb{R}^{N}\right.$ ) and satisfies problem (12) (see [18] for more details). Since $E$ is continuously embedded in $H^{1}\left(\mathbb{R}, \mathbb{R}^{N}\right), u(t) \rightarrow 0$ as $|t| \rightarrow \infty$. The proof is completed.

Lemma 4 Assume that $\left(\mathrm{L}_{1}\right),\left(\mathrm{L}_{2}\right),\left(\mathrm{W}_{1}\right)-\left(\mathrm{W}_{4}\right)$ are satisfied. Then 0 is the only critical point of I such that $I(u)=0$.

Proof By $\left(\mathrm{W}_{1}\right),\left(\mathrm{W}_{2}\right)$, and Lemma 3, we know that 0 is a critical point of $I$ with $I(0)=0$. Now let $u \in E$ be a critical point of $I$ with $I(u)=0$. Then we have

$$
0=2 I(u)-\left\langle I^{\prime}(u), u\right\rangle=-\int_{\mathbb{R}} \widehat{W}(t, u) d t
$$

where $\widehat{W}$ is defined in (4). This together with (ii) of Lemma 2 implies that $|u(t)|=0$ for all $t \in \mathbb{R}$. The proof is completed.

The following lemma is due to Bartsch and Willem [19] and we quote it from [16].

Lemma 5 Let $E$ be a Banach space with a finite-dimensional approximation in the sense that $E=\bigoplus_{j \in \mathbb{N}} E(j)$, where $E(j)$ are all finite-dimensional subspaces. Let $I \in C^{1}(E, \mathbb{R})$ be an even functional and satisfy:

$\left(F_{1}\right)$ For every $k \geq k_{0}$, there exists $R_{k}>0$ such that $I(u) \geq 0$ for every $u \in E_{k}:=\bigoplus_{j \geq k} E(j)$ with $\|u\|=R_{k}$, and $b_{k}:=\inf _{u \in B_{k}} I(u) \rightarrow 0$ as $k \rightarrow \infty$. Here $B_{k}:=\left\{u \in E_{k} \mid\|u\| \leq R_{k}\right\}$.

$\left(\mathrm{F}_{2}\right)$ For every $k \in \mathbb{N}$, there exist $r_{k} \in\left(0, R_{k}\right)$ and $d_{k}<0$ such that $I(u) \leq d_{k}$ for every $u \in$ $E^{k}:=\bigoplus_{j \leq k} E(j)$ with $\|u\|=r_{k}$.

$\left(\mathrm{F}_{3}\right)$ I satisfies $(P S)^{*}$ condition with respect to $\left\{E^{m} \mid m \in \mathbb{N}\right\}$, i.e., every sequence $u_{m} \in E^{m}$ with $I\left(u_{m}\right)<0$ bounded and $\left(\left.I\right|_{E^{m}}\right)^{\prime}\left(u_{m}\right) \rightarrow 0$ as $m \rightarrow \infty$ has a subsequence which converges to a critical point of $I$. 
Then, for each $k \geq k_{0}$, I has a critical value $\xi_{k} \in\left[b_{k}, d_{k}\right]$, hence $\xi_{k}<0$ and $\xi_{k} \rightarrow 0$ as $k \rightarrow \infty$.

Let $E(j)=\operatorname{span}\left\{e_{j}\right\}$ for each $j \in \mathbb{N}$, where $\left\{e_{j}: j \in \mathbb{N}\right\}$ is the system of eigenfunctions given in Remark 2. Now we show that the functional $I$ has the geometric property of Lemma 5 under the conditions of Theorem 1 .

Lemma 6 Assume that $\left(\mathrm{L}_{1}\right),\left(\mathrm{L}_{2}\right),\left(\mathrm{W}_{1}\right)$, and $\left(\mathrm{W}_{2}\right)$ hold. Then there exist a positive integer $k_{0}$ and a sequence $R_{k} \rightarrow 0^{+}$as $k \rightarrow \infty$ such that

$$
\inf _{u \in E_{k},\|u\|=R_{k}} I(u) \geq 0, \quad \forall k \geq k_{0}
$$

and

$$
b_{k}:=\inf _{u \in B_{k}} I(u) \rightarrow 0 \quad \text { as } k \rightarrow \infty
$$

where $E_{k}:=\bigoplus_{j \geq k} E(j)$ and $B_{k}:=\left\{u \in E_{k} \mid\|u\| \leq R_{k}\right\}$ for all $k \in \mathbb{N}$.

Proof Note that $E_{k} \subset E^{+}$for all $k \geq \bar{j}+1$ (see Remark 2 for details). Thus for each $k \geq \bar{j}+1$, by (13) we obtain

$$
\begin{aligned}
I(u) & \geq \frac{1}{2}\|u\|^{2}-\int_{R} \widetilde{W}(t, u) d t \\
& \geq \frac{1}{2}\|u\|^{2}-c_{1}\|u\|_{\theta+1}^{\theta+1}-\beta\|u\|_{p}^{p}, \quad \forall u \in E_{k} .
\end{aligned}
$$

Set

$$
l_{k}=\sup _{u \in E_{k},\|u\|=1}\|u\|_{\theta+1}, \quad \forall k \geq \bar{j}+1
$$

Since $E$ is compactly embedded into $L^{\theta+1}$, we have (see [20])

$$
l_{k} \rightarrow 0^{+} \text {as } k \rightarrow \infty .
$$

For each $k \geq \bar{j}+1$, it follows from (2), (14), (15), and the choice of $\beta$ that

$$
\begin{aligned}
I(u) & \geq \frac{1}{2}\|u\|^{2}-c_{1} l_{k}^{\theta+1}\|u\|^{\theta+1}-\beta \gamma_{p}^{p}\|u\|^{p} \\
& \geq \frac{1}{2}\|u\|^{2}-c_{1} l_{k}^{\theta+1}\|u\|^{\theta+1}-\frac{1}{4}\|u\|^{p}, \quad \forall u \in E_{k} .
\end{aligned}
$$

For each $k \geq \bar{j}+1$, choose

$$
R_{k}=4 c_{1} l_{k}^{\theta+1}
$$

then, by (15),

$$
R_{k} \rightarrow 0^{+} \quad \text { as } k \rightarrow \infty
$$


and hence there exists a positive integer $k_{0} \geq \bar{j}+1$ such that

$$
R_{k}<1, \quad \forall k \geq k_{0}
$$

Now by (17), (18), and (20), we have

$$
\inf _{u \in E_{k},\|u\|=R_{k}} I(u) \geq \frac{1}{2} R_{k}^{2}-\frac{1}{4} R_{k}^{\theta+2}-\frac{1}{4} R_{k}^{p} \geq 0, \quad \forall k \geq k_{0} .
$$

Noting that $I(0)=0$ and

$$
I(u) \geq-c_{1} l_{k}^{\theta+1}\|u\|^{\theta+1}-\frac{1}{4}\|u\|^{p}, \quad \forall k \geq \bar{j}+1, u \in E_{k},
$$

we have

$$
0 \geq \inf _{u \in B_{k}} I(u) \geq-c_{1} l_{k}^{\theta+1} R_{k}^{\theta+1}-\frac{1}{4} R_{k}^{p}, \quad \forall k \geq \bar{j}+1,
$$

which combined with (16) and (19) implies that

$$
b_{k}:=\inf _{u \in B_{k}} I(u) \rightarrow 0 \quad \text { as } k \rightarrow \infty .
$$

The proof is completed.

Lemma 7 Assume that $\left(\mathrm{L}_{1}\right),\left(\mathrm{L}_{2}\right),\left(\mathrm{W}_{1}\right)$, and $\left(\mathrm{W}_{5}\right)$ hold. Then for every $k \in \mathbb{N}$, there exist $r_{k} \in\left(0, R_{k}\right)$ and $d_{k}<0$ such that $I(u) \leq d_{k}$ for every $u \in E^{k}:=\bigoplus_{j \leq k} E(j)$ with $\|u\|=r_{k}$.

Proof For a fixed $k \in \mathbb{N}$, since $E^{k}$ is finite dimensional, there is a constant $C_{k}>0$ such that

$$
C_{k}\|u\|^{\mu} \leq\|u\|_{\mu}^{\mu}, \quad \forall u \in E^{k}
$$

Set $p_{k}=\min \left\{R_{k}, \frac{w_{k}}{\gamma_{\infty}}\right\}$. Then by $\left(\mathrm{W}_{5}\right)$, there exists a constant $0<w_{k}<r$ such that

$$
\widetilde{W}(t, u)=W(t, u) \geq m_{k}|u|^{\mu}, \quad \forall t \in \mathbb{R} \text { and }|u| \leq w_{k},
$$

where $m_{k}=\frac{1}{p_{k}^{\mu-2} C_{k}}$. Now by (2), (21), (22), and Lemma 1, for $u \in E^{k}$ with $\|u\| \leq \frac{w_{k}}{\gamma_{\infty}}$, we get

$$
\begin{aligned}
I(u) & =\frac{1}{2}\left\|u^{+}\right\|^{2}-\frac{1}{2}\left\|u^{-}\right\|^{2}-\int_{\mathbb{R}} \widetilde{W}(t, u) d t \\
& \leq \frac{1}{2}\|u\|^{2}-m_{k}\|u\|_{\mu}^{\mu} \\
& \leq \frac{1}{2}\|u\|^{2}-m_{k} C_{k}\|u\|^{\mu} \\
& =\frac{1}{2}\|u\|^{2}\left(1-\frac{2}{p_{k}^{\mu-2}}\|u\|^{\mu-2}\right) .
\end{aligned}
$$

Choose

$$
0<r_{k}=\left(\frac{2}{3}\right)^{\frac{1}{\mu-2}} p_{k}<p_{k}
$$


and let

$$
d_{k}=-\frac{r_{k}^{2}}{6}<0
$$

If $u \in E^{k}$ with $\|u\|=r_{k}$, we have

$$
I(u) \leq d_{k} .
$$

The proof is completed.

Lemma 8 Assume that $\left(\mathrm{L}_{1}\right),\left(\mathrm{L}_{2}\right),\left(\mathrm{W}_{1}\right),\left(\mathrm{W}_{2}\right)$, and $\left(\mathrm{W}_{4}\right)$ hold. Then I satisfies $(P S)^{*}$ condition with respect to $\left\{E^{m} \mid m \in \mathbb{N}\right\}$.

Proof Let $u_{m} \in E^{m}$ be a $(P S)^{*}$ sequence, that is,

$$
I\left(u_{m}\right) \text { is bounded and }\left(\left.I\right|_{E^{m}}\right)^{\prime}\left(u_{m}\right) \rightarrow 0 \quad \text { as } m \rightarrow \infty .
$$

Then we claim that $\left\{u_{m}\right\}$ is bounded. If not, passing to a subsequence if necessary, we may assume that

$$
\left\|u_{m}\right\| \rightarrow \infty \quad \text { as } m \rightarrow \infty
$$

From (8), (9), (10), we have

$$
\begin{aligned}
2 I\left(u_{m}\right)-\left\langle\left(\left.I\right|_{E^{m}}\right)^{\prime}\left(u_{m}\right), u_{m}\right\rangle & =\int_{\mathbb{R}}\left[\left(\nabla \widetilde{W}\left(t, u_{m}\right), u_{m}\right)-2 \widetilde{W}\left(t, u_{m}\right)\right] d t \\
& \geq(p-2) \beta \int_{\left\{t \in \mathbb{R}|| u_{m}(t) \mid \geq 2 r\right\}}\left|u_{m}\right|^{p} d t
\end{aligned}
$$

for all $m \in \mathbb{N}$. From (23), (24), and (25), it follows that

$$
\frac{\int_{\left\{t \in \mathbb{R} \| u_{m}(t) \mid \geq 2 r\right\}}\left|u_{m}\right|^{p} d t}{\left\|u_{m}\right\|} \rightarrow 0
$$

as $m \rightarrow \infty$. Let

$$
v_{m}(t)= \begin{cases}u_{m}(t), & \text { if }\left|u_{m}(t)\right|<2 r \\ 0, & \text { if }\left|u_{m}(t)\right| \geq 2 r\end{cases}
$$

and

$$
w_{m}(t)=u_{m}(t)-v_{m}(t)
$$

for all $m \in \mathbb{N}$ and all $t \in \mathbb{R}$. By (23), (25), and (28),

$$
c\left(1+\left\|u_{m}\right\|\right) \geq\left\|w_{m}\right\|_{p}^{p}
$$


for all positive integer $m$. From Hölder's inequality, (27), (28), and the equivalence of the norms on the finite-dimensional subspace $E^{-} \oplus E^{0}$, we have

$$
\begin{aligned}
\left\|u_{m}^{-}+u_{m}^{0}\right\|_{2}^{2} & =\left(u_{m}^{-}+u_{m}^{0}, u_{m}\right)_{2} \\
& =\left(u_{m}^{-}+u_{m}^{0}, v_{m}\right)_{2}+\left(u_{m}^{-}+u_{m}^{0}, w_{m}\right)_{2} \\
& \leq\left\|u_{m}^{-}+u_{m}^{0}\right\|_{1}\left\|v_{m}\right\|_{\infty}+\left\|u_{m}^{-}+u_{m}^{0}\right\|_{p^{\prime}}\left\|w_{m}\right\|_{p} \\
& \leq c\left\|u_{m}^{-}+u_{m}^{0}\right\|_{2}\left(1+\left\|w_{m}\right\|_{p}\right)
\end{aligned}
$$

for all $m \in \mathbb{N}$, where $\frac{1}{p}+\frac{1}{p^{\prime}}=1$. Then, from the equivalence of the norms on the finitedimensional subspace $E^{-} \oplus E^{0}$, (29), and (30) it follows that

$$
\begin{aligned}
\left\|u_{m}^{-}+u_{m}^{0}\right\| & \leq c\left\|u_{m}^{-}+u_{m}^{0}\right\|_{2} \\
& \leq c\left(1+\left\|w_{m}\right\|_{p}\right) \\
& \leq c\left(1+\left\|u_{m}\right\|^{\frac{1}{p}}\right)
\end{aligned}
$$

for all $m \in \mathbb{N}$, which implies that

$$
\frac{\left\|u_{m}^{-}+u_{m}^{0}\right\|}{\left\|u_{m}\right\|} \rightarrow 0
$$

as $m \rightarrow \infty$. By (3) we get

$$
|\nabla \widetilde{W}(t, x)| \leq c\left(1+|x|^{p-1}\right), \quad \forall(t, x) \in \mathbb{R} \times \mathbb{R}^{N}
$$

which combined with (2) implies that

$$
\begin{aligned}
\left\langle\left(\left.I\right|_{E^{m}}\right)^{\prime}\left(u_{m}\right), u_{m}^{+}\right\rangle \geq & \left\|u_{m}^{+}\right\|^{2}-\int_{\mathbb{R}}\left|\nabla \widetilde{W}\left(t, u_{m}\right)\right|\left|u_{m}^{+}\right| d t \\
\geq & \left\|u_{m}^{+}\right\|^{2}-c \int_{\mathbb{R}}\left|u_{m}\right|^{p-1}\left|u_{m}^{+}\right| d t-c \int_{\mathbb{R}}\left|u_{m}^{+}\right| d t \\
\geq & \left\|u_{m}^{+}\right\|^{2}-c\left\|u_{m}^{+}\right\|_{\infty} \int_{\left\{t \in \mathbb{R} \| u_{m}(t) \mid \geq 2 r\right\}}\left|u_{m}\right|^{p-1} d t \\
& -c(2 r)^{p-1} \int_{\left\{t \in \mathbb{R} \| u_{m}(t) \mid<2 r\right\}}\left|u_{m}^{+}\right| d t-c\left\|u_{m}^{+}\right\|_{1} \\
\geq & \left\|u_{m}^{+}\right\|^{2}-c\left\|u_{m}^{+}\right\|_{\infty}(2 r)^{-1} \int_{\left\{t \in \mathbb{R} \| u_{m}(t) \mid \geq 2 r\right\}}\left|u_{m}\right|^{p} d t \\
& -c(2 r)^{p-1}\left\|u_{m}^{+}\right\|_{1}-c\left\|u_{m}^{+}\right\|_{1}\left|u_{m}\right|^{p} d t \\
\geq & \left\|u_{m}^{+}\right\|^{2}-c \gamma_{\infty}\left\|u_{m}^{+}\right\|(2 r)^{-1} \int_{\left\{t \in \mathbb{R} \| u_{m}(t) \mid \geq 2 r\right\}} \\
& -c(2 r)^{p-1} \gamma_{1}\left\|u_{m}^{+}\right\|-c \gamma_{1}\left\|u_{m}^{+}\right\| .
\end{aligned}
$$

From this and (26) it follows that

$$
\frac{\left\|u_{m}^{+}\right\|}{\left\|u_{m}\right\|} \rightarrow 0
$$


as $m \rightarrow \infty$. Combining (31) and (32), one gets

$$
1=\frac{\left\|u_{m}\right\|}{\left\|u_{m}\right\|} \leq \frac{\left\|u_{m}^{+}\right\|+\left\|u_{m}^{-}+u_{m}^{0}\right\|}{\left\|u_{m}\right\|} \rightarrow 0
$$

as $m \rightarrow \infty$, which is a contradiction. Hence $\left\{u_{m}\right\}$ is bounded. Noting that by Lemma $3, I^{\prime}$ is a compact perturbation of the identity in $E \ominus E^{0}$. Since $E^{0}$ has finite dimension, $\left\{u_{m}\right\}$ has a subsequence converging to a critical point of $I$ (see [18]). Hence, $I$ satisfies the $(P S)^{*}$ condition. The proof is completed.

Proof of Theorem 1 It follows from Lemmas 6-8 that the functional $I$ satisfies the conditions $\left(\mathrm{F}_{1}\right)-\left(\mathrm{F}_{3}\right)$ of Lemma 5 . Therefore, by Lemma 5 , there exists a sequence of critical values $\xi_{k}<0$ with $\xi_{k} \rightarrow 0$ as $k \rightarrow \infty$. Let $\left\{u_{k}\right\}$ be a sequence of critical points of $I$ corresponding to these critical values, i.e., $I\left(u_{k}\right)=\xi_{k}$ and $I^{\prime}\left(u_{k}\right)=0$ for all $k$. Then by Lemma 3, $\left\{u_{k}\right\} \subset C^{2}\left(\mathbb{R}, \mathbb{R}^{N}\right)$ is a sequence of homoclinic solutions of problem (12). Moreover, $\left\{u_{k}\right\}$ forms a $(P S)$ sequence in $E$. By Lemma 8 and Remark 3.19 in [20], I satisfies the $(P S)$ condition and hence we may assume without loss of generality that $u_{k} \rightarrow u$ in $E$ as $k \rightarrow \infty$. Evidently, $u$ is a critical point of $I$ with $I(u)=0$. Then by Lemma $4, u$ must be 0 . Thus $u_{k} \rightarrow 0$ in $E$ as $k \rightarrow \infty$. By (2), we further have $u_{k} \rightarrow 0$ in $L^{\infty}\left(\mathbb{R}, \mathbb{R}^{N}\right)$ as $k \rightarrow \infty$. Therefore, for $k$ large enough, they are homoclinic solutions of problem (1). The proof is completed.

\section{Competing interests}

The author declares that they have no competing interests.

\section{Acknowledgements}

This research was supported by National Natural Science Foundation of China (No. 11426190).

Received: 22 April 2015 Accepted: 18 November 2015 Published online: 26 January 2016

\section{References}

1. Alves, $\mathrm{CO}$, Carrião, PC, Miyagaki, $\mathrm{OH}$ : Existence of homoclinic orbits for asymptotically periodic systems involving Duffing-like equation. Appl. Math. Lett. 16, 639-642 (2003)

2. Coti-Zelati, V, Rabinowitz, PH: Homoclinic orbits for second order Hamiltonian systems possessing superquadratic potentials. J. Am. Math. Soc. 4, 693-727 (1991)

3. Ding, YH: Existence and multiplicity results for homoclinic solutions to a class of Hamiltonian systems. Nonlinear Anal. 25, 1095-1113 (1995)

4. Daouas, A: Homoclinic orbits for superquadratic Hamiltonian systems without a periodicity assumption. Nonlinear Anal. 74, 3407-3418 (2011)

5. Izydorek, M, Janczewska, J: Homoclinic solutions for nonautonomous second order Hamiltonian systems with a coercive potential. J. Math. Anal. Appl. 335, 1119-1127 (2007)

6. LV, X, Jiang, J: Existence of homoclinic solutions for a class of second-order Hamiltonian systems with general potentials. Nonlinear Anal. 13, 1152-1158 (2012)

7. Rabinowitz, PH, Tanaka, K: Some results on connecting orbits for a class of Hamiltonian systems. Math. Z. 206, 473-499 (1990)

8. Sun, J, Wu, T: Multiplicity and concentration of homoclinic solutions for some second order Hamiltonian system. Nonlinear Anal. 114, 105-115 (2015)

9. Wu, SP, Yang, HT: A note on homoclinic orbits for second order Hamiltonian system. Appl. Math. J. Chin. Univ. Ser. B 13, 251-262 (1998)

10. Wan, LL, Tang, CL: Existence and multiplicity of homoclinic orbits for second order Hamiltonian systems without (AR) condition. Discrete Contin. Dyn. Syst. 15, 255-271 (2011)

11. Yang, J, Zhang, FB: Infinitely many homoclinic orbits for the second-order Hamiltonian systems with super-quadratic potentials. Nonlinear Anal. 10, 1417-1423 (2009)

12. Yang, MH, Han, ZQ: Infinitely many homoclinic solutions for second-order Hamiltonian systems with odd nonlinearities. Nonlinear Anal. 74, 2635-2646 (2011)

13. Ye, Y, Tang, CL: Multiple homoclinic solutions for second-order perturbed Hamiltonian systems. Stud. Appl. Math $132,112-137$ (2014)

14. Zou, WM, Li, SJ: Infinitely many homoclinic orbits for the second-order Hamiltonian systems. Appl. Math. Lett. 16 1283-1287 (2003)

15. Zhang, Z, Xiang, T, Yuan, R: Homoclinic solutions for subquadratic Hamiltonian systems without coercive conditions Taiwan. J. Math. 18, 1089-1105 (2014) 
16. Zhang, QY, Chu, LP: Homoclinic solutions for a class of second order Hamiltonian systems with locally defined potentials. Nonlinear Anal. 75, 3188-3197 (2012)

17. Kato, T: Perturbation Theory for Linear Operators. Springer, Berlin (1995)

18. Mawhin, J, Willem, M: Critical Point Theory and Hamiltonian Systems. Springer, New York (1989)

19. Bartsch, T, Willem, M: On an elliptic equation with concave and convex nonlinearities. Proc. Am. Math. Soc. 123, 3555-3561 (1995)

20. Willem, M: Minimax Theorems. Birkhäuser, Boston (1996)

Submit your manuscript to a SpringerOpen ${ }^{\odot}$ journal and benefit from:

- Convenient online submission

Rigorous peer review

- Immediate publication on acceptance

- Open access: articles freely available online

- High visibility within the field

- Retaining the copyright to your article 